\title{
Closed Form Expressions for Scattering Matrix of Simple Targets in Multilayer Structures
}

\author{
Sidnei J. S. Sant'Anna ${ }^{(1,2)}$ \\ Divisão de Processamento de Imagens \\ ${ }^{(1)}$ Instituto Nacional de Pesquisas Espaciais (INPE) \\ São José dos Campos, SP, Brazil \\ sidnei@dpi.inpe.br
}

\author{
J. C. da S. Lacava ${ }^{(2)}$ and David Fernandes ${ }^{(2)}$ \\ Laboratório de Antenas e Propagação \\ ${ }^{(2)}$ Instituto Tecnológico de Aeronáutica (ITA) \\ São José dos Campos, SP, Brazil \\ \{lacava,david\}@ita.br
}

\begin{abstract}
Scattering matrix of a simple target embedded in multilayer planar structure is derived. The structure is excited by an elliptically polarized plane wave incident at an oblique angle. The calculation of the electromagnetic fields is performed in spectral domain. The scattering matrix of a planar electric dipole printed on upper interface and surrounding by free-space is evaluated. The obtained results agree with those existing on the literature for normal incidence case.
\end{abstract}

Keywords-component; scattering matrix; full-wave technique, SAR polarimetry

\section{INTRODUCTION}

The retrieval of natural target parameters from microwave remote sensing data has been subject to numerous studies. The understanding of electromagnetic target reflective properties is the key for a correct data interpretation. In general, these reflective properties are described by a matrix, called scattering matrix. The scattering matrix syntheses both polarimetric and electromagnetic properties of a target, providing the far electric field scattered in a specific direction as a response to an electromagnetic wave incident on the target. Therefore, the incident and the scattering directions must be specified. In this way, a target is completely characterized by its scattering matrix, which contains all information about scattering process.

From polarimetric SAR images and using the knowledge of scattering matrix it is possible, for instance, to develop mathematical models for natural targets and to derive several polarimetric target descriptors, like the polarimetric response, the radar cross section, the entropy, the anisotropy, the terrain azimuth slope, among others. It is also possible to improve the classification accuracy of land cover and use, to identify the scattering mechanisms intrinsic on an ensemble of pixels, and to built up a sensor calibration processes. In this fashion, the computation of scattering matrix plays a central rule in the radar remote sensing imagery.

In this work the determination of the scattering matrix of multilayer planar structures is addressed. An elliptically polarized plane wave with oblique incidence is employed as the excitation mechanism. In Section II, a general theory for computation of the electromagnetic fields presenting in the structure is developed. A simple structure is then used to evaluate the scattering matrix. Finally, the scattering matrix elements are established (Section III) in a simple and closed form for any incident and scattering directions. A comparison for normal directions case is also made.

\section{GENERAL THEORY}

The structure in analysis is composed by $N+2$ isotropic, linear and homogenous layers stacked in $z$ direction. The geometry of the planar structure is depicted on Fig. 1. The layers are assumed to be infinite along $x$ and $y$ directions. The lower layer occupies the $z$-negative region and is called by ground layer. It has complex permittivity $\varepsilon_{g}$ and complex permeability $\mu_{g}$. The $N$ layers are characterized by thickness $\ell_{n}$, complex permittivity $\varepsilon_{n}$ and complex permeability $\mu_{n}$, where $1 \leq n \leq N$. The planar interface $z=d_{N}$ separates the $N$ th layer from the free-space region (upper layer). Metallic patches, which will act as scatter elements, are printed on each $N+1$ layer interfaces. The patches are perfect conducting surfaces having infinitesimal thickness. The development is based on global right handed rectangular coordinate system that is located at the top of ground layer (interface $z=0$ ) lying on $x y$-plane.

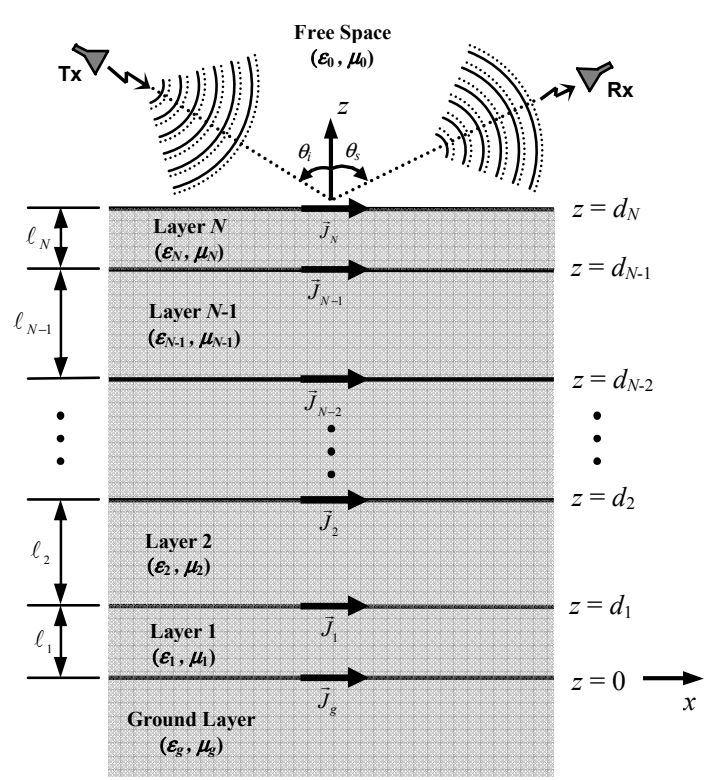

Figure 1. Geometry of the planar structure with $N+2$ layers. 
According to the methodology developed in [1] the structure is treated as a boundary value problem. The analysis is carried out by employing the spectral domain full-wave technique. The induced electric surface current densities located on the metallic patches are the virtual sources of the scattered fields. Firstly, the wave equations for each layer are solved in the Fourier domain. The wave equations for the $m$ th layer, in source-free medium, is written by:

$$
\begin{gathered}
\nabla^{2} \vec{E}_{m}(x, y, z)+k_{m}^{2} \vec{E}_{m}(x, y, z)=0, \\
\nabla^{2} \vec{H}_{m}(x, y, z)+k_{m}^{2} \vec{H}_{m}(x, y, z)=0,
\end{gathered}
$$

where $k_{m}^{2}=\omega^{2} \mu_{m} \varepsilon_{m}, k_{m}$ is the wave number in the layer $m, \omega$ is the angular frequency and the vectors $\vec{E}_{m}(x, y, z)$ and $\vec{H}_{m}(x, y, z)$ are the complex electric and magnetic fields, respectively. The time harmonic variation of the fields is represented by $e^{i \omega t}$. For the free-space region and ground layer the index $m$ is equal to 0 and $g$, respectively.

Applying the double Fourier transform to (1) and (2) yields a differential equation system. To solve the system the field components, in spectral domain, are assumed to be:

$$
\begin{aligned}
& \mathcal{E}_{m \eta}\left(k_{x}, k_{y}, z\right)=\mathfrak{e}_{m \eta}\left(k_{x}, k_{y}\right) e^{i \gamma_{m} z}, \\
& \mathcal{H}_{m \eta}\left(k_{x}, k_{y}, z\right)=\mathfrak{h}_{m \eta}\left(k_{x}, k_{y}\right) e^{i \gamma_{m} z},
\end{aligned}
$$

with

$$
\gamma_{m}=(-1)^{\tau} \sqrt{k_{m}^{2}-k_{x}^{2}-k_{y}^{2}}, \quad \operatorname{Im}\left\{\gamma_{m}\right\} \leq 0,
$$

where $\mathbb{e}_{m \eta}\left(k_{x}, k_{y}\right)$ and $\mathbb{h}_{m \eta}\left(k_{x}, k_{y}\right)$ are the amplitude of the transformed field components, $k_{x}$ and $k_{y}$ are the spectral variables, $\gamma_{m}$ is the propagation constant in the layer $m$, $\eta=x, y$ or $z$ and $\operatorname{Im}\{\cdot\}$ means the imaginary part function. The $\tau$ variable defines the wave propagation direction, assuming values 1 or 2 . The former value is gotten in the upper layer (free-space region), representing propagation in positive $z$-direction. On the otherwise, for the ground layer $\tau$ is equal to 2 , i.e., a wave propagating in negative $z$-direction. However, both values are accepted by $\tau$ for the inner layers.

The inverse Fourier transform provides the electromagnetic fields $\vec{E}_{m}(x, y, z)$ and $\vec{H}_{m}(x, y, z)$ in spatial domain. Employing the inverse transformation as defined by (6) and (7), the field components are obtained:

$$
E_{m \eta}(x, y, z)=\frac{1}{4 \pi^{2}} \int_{-\infty}^{+\infty} \int_{-\infty} \mathbb{e}_{m \eta}\left(k_{x}, k_{y}\right) e^{-i\left(k_{x} x+k_{y} y+\gamma_{m} z\right)} d k_{x} d k_{y},
$$

$$
H_{m \eta}(x, y, z)=\frac{1}{4 \pi^{2}} \int_{-\infty}^{+\infty} \int_{-\infty} \mathfrak{h}_{m \eta}\left(k_{x}, k_{y}\right) e^{-i\left(k_{x} x+k_{y} y+\gamma_{m} z\right)} d k_{x} d k_{y}
$$

Interesting relations among the amplitudes of the transformed fields are derived by introducing (6) and (7) in the Maxwell's curl equations. In these relations the amplitudes of the transversal components ( $x$ and $y$ directions) are written in functions of the amplitude of the longitudinal ones ( $z$ direction). After that, the boundary conditions for the electromagnetic fields are applied at each interface. This approach yields a set of $4 N+4$ equations with equal numbers of unknowns. The analytical solution of this system leads to the spectral Green's functions in a closed and simple form. The Green's functions jointly with the transformed superficial density currents allow the determination of the transformed fields at any point of the multilayer structure. In the free-space region, for example, the longitudinal transformed electromagnetic field components are expressed by:

$$
\begin{aligned}
\mathbb{e}_{0 z}\left(k_{x}, k_{y}\right)= & \sum_{u} G_{z u x}^{(m)}\left(k_{x}, k_{y}, d_{u}\right) j_{u x}\left(k_{x}, k_{y}\right)+ \\
& \sum_{u} G_{z u y}^{(m)}\left(k_{x}, k_{y}, d_{u}\right) j_{u y}\left(k_{x}, k_{y}\right), \\
\mathbb{h}_{0 z}\left(k_{x}, k_{y}\right)= & \sum_{u} Q_{z u x}^{(m)}\left(k_{x}, k_{y}, d_{u}\right) j_{u x}\left(k_{x}, k_{y}\right)+ \\
& \sum_{u} Q_{z u y}^{(m)}\left(k_{x}, k_{y}, d_{u}\right) j_{u y}\left(k_{x}, k_{y}\right),
\end{aligned}
$$

where $G_{z u v}^{(m)}\left(k_{x}, k_{y}, d_{u}\right)$ and $Q_{z u v}^{(m)}\left(k_{x}, k_{y}, d_{u}\right)$ represent, respectively, the electrical and magnetic spectral Green's functions in the $m$ th layer, which relate the $v(v=x$ or $y)$ components of the electric and magnetic fields to the transformed superficial density current $j_{u v}\left(k_{x}, k_{y}\right)$ located on interface $d_{u}$ and $u \in\{g, 1,2, \ldots, N\}$ defines the interface, with $d_{g}=0$.

In order to calculate the far electromagnetic fields scattered by the multilayer structure asymptotic expressions are derived. The stationary phase method $[1,2]$ is used to obtain these expressions. From the asymptotic expressions and knowing the current densities, the scattering matrix elements are completely determined. If necessary the method of moments, for instance, could be applied to determine the current densities induced on the metallic patches. The electric far field, using the stationary phase method is given by:

$$
\vec{E}_{0}(r, \theta, \phi) \cong \mathbb{C}\left\{\hat{\theta} \mathbb{e}_{0 z}\left(k_{x e}, k_{y e}\right)-\hat{\phi} \eta_{0} \mathfrak{h}_{0 z}\left(k_{x e}, k_{y e}\right)\right\},
$$

in the spherical coordinate system, where the intrinsic impedance of free-space is represented by $\eta_{0}, k_{x e}=k_{0} \sin \theta \cos \phi$ and $k_{y e}=k_{0} \sin \theta \operatorname{sen} \phi$ are the stationary phase points and $\mathbb{C}=-\frac{i k_{0}}{2 \pi} \frac{e^{-i k_{0} r}}{r} \cot \theta$, with $r$ being the distance between the receiving antenna and the target and $k_{0}$ representing the wave 
number of the exciting wave. The electric far field will be used to calculate the scattering matrix elements in Section III.

\section{A. Four Layers Structure}

The formulation is now applied to a structure containing four layers $(N=2)$. A planar electric dipole having any orientation in $x y$-plane is printed on interface $z=d_{2}$. For this structure, the transformed electric far field components in the free-space region are given by:

$$
\begin{aligned}
& \mathbb{e}_{0 z}\left(k_{x}, k_{y}\right)=\frac{4 \omega^{2} \Omega_{0}^{e}}{\Delta_{e}}\left\{k_{x} j_{2 x}\left(k_{x e}, k_{y e}\right)+k_{y} j_{2 y}\left(k_{x e}, k_{y e}\right)\right\},(11 \\
& \mathfrak{h}_{0 z}\left(k_{x}, k_{y}\right)=\frac{4 \omega^{2} \Omega_{0}^{h}}{\Delta_{m}}\left\{k_{y} j_{2 x}\left(k_{x e}, k_{y e}\right)-k_{x} j_{2 y}\left(k_{x e}, k_{y e}\right)\right\},(12
\end{aligned}
$$

where the following factors properly grouped together with the spectral variables portray the spectral Green's functions of the free-space.

$$
\begin{array}{r}
\Omega_{0}^{e}=\gamma_{2}\left\{\varepsilon_{1} \gamma_{2}\left(\varepsilon_{1} \gamma_{g} \sin \alpha_{1}-i \varepsilon_{g} \gamma_{1} \cos \alpha_{1}\right) \sin \alpha-\right. \\
\left.\varepsilon_{2} \gamma_{1}\left(\varepsilon_{1} \gamma_{g} \cos \alpha_{1}+i \varepsilon_{g} \gamma_{1} \sin \alpha_{1}\right) \cos \alpha\right\}
\end{array}
$$

$$
\begin{array}{r}
\Omega_{0}^{h}=\omega \mu_{2}\left\{\mu_{1} \gamma_{2}\left(\mu_{g} \gamma_{1} \cos \alpha_{1}+i \mu_{1} \gamma_{g} \sin \alpha_{1}\right) \cos \alpha-\right. \\
\left.\mu_{2} \gamma_{1}\left(\mu_{g} \gamma_{1} \sin \alpha_{1}-i \mu_{1} \gamma_{g} \cos \alpha_{1}\right) \sin \alpha\right\}
\end{array}
$$

$$
\begin{aligned}
& \Delta_{e}=-4 \omega^{3} e^{-i \alpha_{0}} \\
& \left\{\varepsilon_{1} \gamma_{2}\left(\varepsilon_{2} \gamma_{0} \cos \alpha+i \varepsilon_{0} \gamma_{2} \sin \alpha\right)\left(\varepsilon_{g} \gamma_{1} \cos \alpha_{1}+i \varepsilon_{1} \gamma_{g} \sin \alpha_{1}\right)+\right. \\
& \left.\varepsilon_{2} \gamma_{1}\left(\varepsilon_{0} \gamma_{2} \cos \alpha+i \varepsilon_{2} \gamma_{0} \sin \alpha\right)\left(\varepsilon_{1} \gamma_{g} \cos \alpha_{1}+i \varepsilon_{g} \gamma_{1} \sin \alpha_{1}\right)\right\}
\end{aligned}
$$

$\Delta_{m}=4 \omega^{3} e^{-i \alpha_{0}}$

$\left\{\mu_{1} \gamma_{2}\left(\mu_{2} \gamma_{0} \cos \alpha+i \mu_{0} \gamma_{2} \sin \alpha\right)\left(\mu_{g} \gamma_{1} \cos \alpha_{1}+i \mu_{1} \gamma_{g} \sin \alpha_{1}\right)+\right.$

$\left.\mu_{2} \gamma_{1}\left(\mu_{0} \gamma_{2} \cos \alpha+i \mu_{2} \gamma_{0} \sin \alpha\right)\left(\mu_{1} \gamma_{g} \cos \alpha_{1}+i \mu_{g} \gamma_{1} \sin \alpha_{1}\right)\right\},(16)$

$\alpha_{0}=\gamma_{0} d_{2}, \quad \alpha_{1}=\gamma_{1} d_{1}, \quad \alpha_{2}=\gamma_{2} d_{2}, \quad \alpha_{3}=\gamma_{2} d_{1} \quad$ and $\alpha=\alpha_{2}-\alpha_{3}$. Notice that only the components $\mathfrak{e}_{0 z}\left(k_{x}, k_{y}\right)$ and $\mathrm{h}_{0 z}\left(k_{x}, k_{y}\right)$ are necessary to compute the electric far field, as shown in (10).

\section{SCATTERING MATRIX}

Considering a target excited by an electromagnetic plane wave, at the target the electric field of the incident wave can be written, in standard spherical coordinate system, as

$$
\vec{E}^{i}=\hat{\theta} E_{\theta}^{i}+\hat{\phi} E_{\phi}^{i}
$$

where $E_{\theta}^{i}$ and $E_{\phi}^{i}$ are the complex amplitudes of the components of incident electric field, in $\theta$ and $\phi$ directions, respectively. The amplitude $E_{r}^{i}$ is zero since the incident wave is supposed to be plane.

The wave defined by this electric field will set up currents in the scatter, which in turn reradiate a scattered wave. In the far zone of the target, the scattered wave is an outgoing, spherical wave, which can be approximated by a plane wave over the relatively small area occupied by the receiving antenna [3].

The scattering process can be modeled as a linear transformation between the incident and the scattered electric fields, given by:

$$
\left[\begin{array}{c}
E_{\theta}^{s} \\
E_{\phi}^{s}
\end{array}\right]=\frac{e^{-i k_{0} r}}{r}\left[\begin{array}{ll}
S_{\theta \theta} & S_{\theta \phi} \\
S_{\phi \theta} & S_{\phi \phi}
\end{array}\right]\left[\begin{array}{c}
E_{\theta}^{i} \\
E_{\phi}^{i}
\end{array}\right],
$$

where $r$ and $k_{0}$ are defined in the same way that in (10). This transformation is characterized by the scattering matrix, which is $2 \times 2$ complex matrix and is denoted by $[S]$. The $[S]$ matrix describes completely the interaction process between an electromagnetic wave and a target. Its elements are dependent of operating frequency, electromagnetic properties, target shape and orientation, illuminating angles and they are dimensionless. The diagonal elements $\left(S_{\theta \theta}\right.$ and $\left.S_{\phi \phi}\right)$ and the off-diagonal ones are called respectively co- and crosspolarized components.

According to [3], the electric field of a plane wave traveling in the direction $\hat{k}$ could be characterized in terms of horizontal $\hat{h}$ and vertical $\hat{v}$ linear polarization components, defining a coordinate system $(\hat{k}, \hat{v}, \hat{h})$. Furthermore this system is chosen to coincide with $(\hat{r}, \hat{\theta}, \hat{\phi})$ in a standard spherical coordinate system. Therefore, from (17), a linear vertical polarized incident wave is generated by assigning $E_{\phi}^{i}=0 \mathrm{~V} / \mathrm{m}$. If a target is illuminated by this wave, the electric field components of the scattered wave can be found by using (18). The expressions of these two components have a dependency with respect to $E_{\theta}^{i}$, which can be cancelled doing $E_{\theta}^{i}=1 \mathrm{~V} / \mathrm{m}$. After normalizing the elements of scattering matrix are directly related to the components of the scattered electric field $\left(S_{\theta \theta}=E_{\theta}^{s}\right.$ and $S_{\phi \theta}=E_{\phi}^{s}$ ). In a similar way $S_{\theta \phi}=E_{\theta}^{s}$ and $S_{\phi \phi}=E_{\phi}^{s}$ are the components of a scattered electric field when the incident wave is horizontal linearly polarized $\left(E_{\theta}^{i}=0 \mathrm{~V} / \mathrm{m}\right.$ and $\left.E_{\phi}^{i}=1 \mathrm{~V} / \mathrm{m}\right)$.

Applying this approach to the four layers structure, the scattered electric field is obtained by using (10), (11) and (12). The components of the scattered electric field for linear vertical and horizontal polarized incident wave are given by:

$$
\begin{gathered}
E_{\theta}^{s(v, h)}\left(r, \theta_{s}, \phi_{s}\right)=\mathbb{C} \mathbb{e}_{0 z}^{(v, h)}\left(k_{x e}, k_{y e}\right), \\
E_{\phi}^{s(v, h)}\left(r, \theta_{s}, \phi_{s}\right)=-\mathbb{C} \eta_{0} \mathfrak{h}_{0 z}^{(v, h)}\left(k_{x e}, k_{y e}\right),
\end{gathered}
$$


where the superscripts $(v, h)$ are associated to incident wave polarization.

Note that the induced current on the metallic patch depends on the incident wave polarization. In order to search for a relation between the induced currents on the patch due to each linear polarization, the reciprocity theorem is applied, resulting

$$
\mathbf{e}_{0 z}^{(h)}\left(k_{x e}, k_{y e}\right)=\eta_{0} \mathfrak{h}_{0 z}^{(v)}\left(k_{x e}, k_{y e}\right)
$$

For a planar dipole oriented along of $x$ axis of a standard rectangular coordinate system the component of the current density along of $y$ axis can be neglected. Then using (11), (12) and (21) the above mentioned induced currents are related to

$$
j_{2 x}^{(h)}\left(k_{x e}, k_{y e}\right)=-\mathbb{C}_{1} \tan \phi_{s} j_{2 x}^{(v)}\left(k_{x e}, k_{y e}\right),
$$

where the constant $\mathbb{C}_{1}$ is expressed by:

$$
\mathbb{C}_{1}=\frac{\Delta_{e} \Omega_{0}^{h}}{\Delta_{m} \Omega_{0}^{e}} \eta_{0}
$$

Substituting (22) in (19) and (20), the following scattering matrix is established for any incident and scattering directions

$$
[S]=\mathbb{C}_{2}\left[\begin{array}{cc}
\cos ^{2} \phi_{s} / \mathbb{C}_{1} & -\sin \phi_{s} \cos \phi_{s} \\
-\sin \phi_{s} \cos \phi_{s} & \mathbb{C}_{1} \sin ^{2} \phi_{s}
\end{array}\right],
$$

where $\mathbb{C}_{2}$ is a proportionality constants given by:

$$
\mathbb{C}_{2}=-\frac{i 2 \omega^{2} k_{0}^{2} \eta_{0} \Omega_{0}^{h}}{\pi \Delta_{m}} \cos \theta_{s} j_{2 x}^{(v)}\left(k_{x e}, k_{y e}\right) .
$$

In a particular case, set out by normal incidence direction $\left(\theta_{s}=0^{\circ}\right)$ and when all layers are characterized by the same parameters of free-space, i.e. permittivity $\varepsilon_{0}$ and permeability $\mu_{0}$, the scattering matrix becomes:

$$
[S]=\mathbb{C}_{2}\left[\begin{array}{cc}
\cos ^{2} \phi_{s} & -\sin \phi_{s} \cos \phi_{s} \\
-\sin \phi_{s} \cos \phi_{s} & \sin ^{2} \phi_{s}
\end{array}\right]
$$

The expression (26) is similar to that showed in [3] for a filamentary dipole for backscatter direction in BSA convention, unless the constants. As stated in [3] the scattering matrix, for normal incidence, is expressed in terms of the $\alpha$ angle between the axis of the dipole and the vector basis $\hat{h}_{i}$. The $\alpha$ angle and the $\phi_{s}$ angle, in our formulation, are related by $\alpha=90^{\circ}-\phi_{s}$.

\section{CONCLUSION}

In this work was determined an approach in spectral domain to derive the scattering matrix of multilayer planar structures. The structure was excited by an elliptically polarized plane wave having oblique incidence. The scattering matrix components were established by employing this approach to a simple structure. The structure contains four layers $(N=2)$ and a planar electric dipole printed on the interface between the $N$ th layer and the free-space region. The obtained results for a particular case (normal incidence and the dipole surrounded by the freespace) are in accordance with those presented on the specialized literature for normal incidence case.

\section{REFERENCES}

[1] J.C.S. Lacava, "O emprego da transformada dupla de Fourier em problemas de aberturas planas e na análise de antenas de microlinha". PhD Thesis (in portuguese), ITA, 1985.

[2] C.A. Balanis, Antenna theory: analysis and design. New York, John Willey \& Sons Inc., 1997. 941p.

[3] F.T. Ulaby, C. Elachi, Radar polarimetry for geoscience applications. Norwood, Artech House, 1990. 364p. 